\title{
Functional Electrical Stimulation: Current Status and Future Prospects of Applications to the Neuromuscular System in Spinal Cord Injury
}

\author{
P. Hunter Peckham, Ph.D. \\ Departments of Biomedical Engineering and Orthopaedics, Highland View Hospital; \\ Spinal Injury Center, Case Western Reserve University and Veterans Administra- \\ tion Medical Center, Cleveland, Ohio U.S.A.
}

\begin{abstract}
Summary
Functional electrical stimulation (FES) is a means of eliciting activation of the nervous system in order to elicit either a therapeutic or functional effect. The results of research performed over the past 10 years has provided a scientific knowledge base for clinical studies. The results of clinical studies demonstrate the viability of utilising FES to restore function to the spinal injured individual. This article focuses on applications to the neuromuscular system which are relevant to the care of these individuals.
\end{abstract}

\section{Introduction}

Functional electrical stimulation (FES) is a technique of eliciting controlled neural activation through the application of low levels of electrical current. The objective of FES is to obtain responses in the nervous system to achieve movement or sensation. In the former case, the desired purpose is to elicit a motor response (muscle contraction) through activation of a specific group of nerve fibres, typically using fibres of peripheral nerves. This may be achieved by activation of motor efferent or through reflex pathways by activation of sensory afferent nerve fibres. The technique may also include activation of sensory nerve fibres for the purpose of eliciting a perceived response in the skin which may be used to provide sensory cues to the asensory extremity.

Functional electrical stimulation includes both therapeutic applications and applications to obtain functional restoration of movement. Therapeutic applications include those purposes which are employed to halt or reverse the progress of a disabling condition. Generally, the time period for these applications is relatively short lived (maximal periods of several years). Functional restoration of movement includes the applications to enhance manipulation in the upper extremity or locomotion in the lower extremity. These applications generally are expected to be utilised for the lifetime of the user, since the state 
of the neurological injury generally is stable at the onset of the implementation and remains so despite the intervention. Other applications of FES which are outside the scope of this paper include activation of the diaphragm for respiratory pacing and control of the urinary bladder.

\section{Methods}

Electrical stimulation is delivered to the tissue via electrodes which may be placed either on the skin surface, or subcutaneously. Skin surface electrodes are generally used in therapeutic stimulation, and have undergone significant development. Electrodes are now commercially available which provide excellent electrical contact to the skin, and may be applied without removal or irritation, for days. The general availability of such electrodes, as well as the availability of external stimulator devices, has made clinical studies of therapeutic uses of electrical stimulation quite common.

Subcutaneous electrodes generally have been used for functional restoration. Electrodes of this type may either be placed in or on the muscle, known as intramuscular or epimysial, or around or adjacent to the nerve. In all cases, the stimulation elicits contraction of the muscle primarily through activation of peripheral afferent nerve fibres, rather than by direct muscle excitation. In our laboratories, intramuscular electrodes are inserted percutaneously for periods of months to years providing an excellent means of eliciting a focused contraction without surgery. The electrode (Fig. 1), which is coiled into a helical configuration, is introduced through the skin using a 19 gauge hypodermic needle. The complications of this technique are few, and include breakage, skin

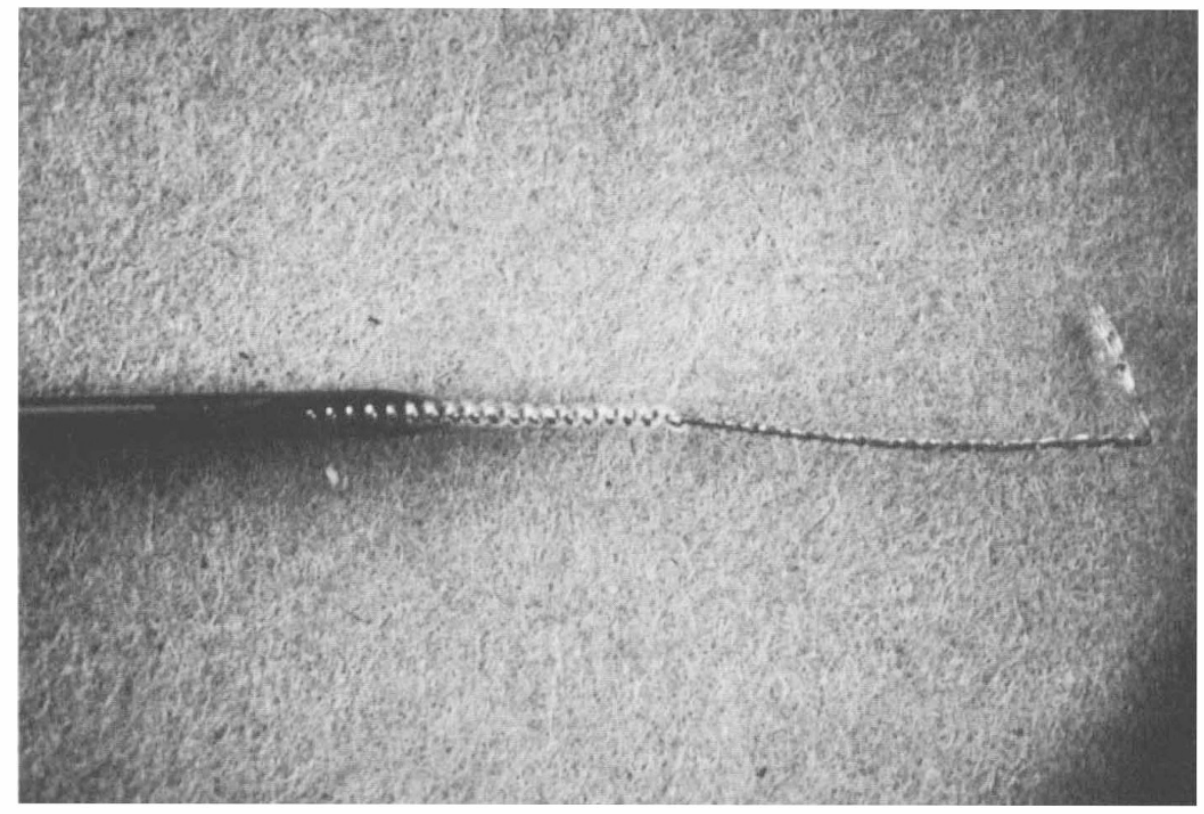

Figure 1 Intramuscular electrode used for chronic percutaneous implantation. The connector assembly provides electrical access to the electrodes and protection of the implantation site. 
irritation and possible infection, although only one electrode of several thousand implanted has become infected (Marsolais, 1986). This approach has proven to be an ideal means to enable development of quite sophisticated and complex clinical systems for manipulation and locomotion without subjecting the patient to surgical intervention for electrode or stimulator implantation.

\section{Applications of electrical stimulation}

\section{Therapeutic applications}

Electrical stimulation has been employed for a variety of therapeutic purposes in the musculoskeletal system. These applications include muscle strengthening, relief of spasticity, reversal of joint contracture and muscle adhesions, and correction of spinal curvature.

Muscles which are atrophied due to disuse have been demonstrated to increase force and fatigue resistance in response to electrical activation. The alterations in force and fatigue resistance are accompanied by a change in the metabolic profile of the muscle. Assuming a chronic, low frequency (on the order of 10 $\mathrm{Hz}$ ) application of stimuli, the atrophied type II muscle fibres of the disuse atrophied muscle will change toward type I fibre which is larger in diameter (Mortimer, 1981). While optimal paradigms of activity have not been identified, the changes that are induced are used to advantage in the case of muscle weakness. Examples of this approach are in maintaining muscle strength during immobilisation for fracture, restoration of voluntary function in paretic muscles, and preventing joint subluxation in stroke. In spinal cord injury, muscle stimulation has been reported to improve blood flow through the muscle, thus decreasing stasis and the likelihood of pulmonary embolus. This should be particularly relevant in cases of prolonged immobilisation.

The use of electrical stimulation for muscle strengthening is an essential element of functional activation of totally paralysed muscles as well. Muscles that are unable to generate and sustain satisfactory performance prior to the conditioning stimulation can thus be made to alter their metabolism to perform at levels which are sufficient for clinical usage. Subjects have reported satisfaction with resultant cosmetic benefit of increased girth which results from the muscle hypertrophy, which many feel improves appearance. Virtually every investigator involved in utilising electrical stimulation for restoration of functional movement incorporates a muscle strengthening program in the clinical protocol.

\section{Functional applications}

A second major area of FES concerns applications to restore movement and control, which is sometimes referred to as functional neuromuscular stimulation, or FNS. This area is primarily experimental at present, although clinical devices are provided on individual bases in some centres. Major advances have been made in both technological development and clinical applications.

FNS systems involve interaction between the user, the device he uses to deliver the stimulus, and the action generated. Thus, he must perform some action to control the device, which then generates the appropriate stimulus patterns which are applied to the muscles to move the extremity. For FNS 
applications, most systems utilise four or more channels of stimulation. The stimulus generated is regulated by the action of the user, thus requiring the presence of an adequate source for generation of this command control signal. Systems of this type are known as open loop systems, since the only alterations in the action of the device are those imposed by the user. Open loop systems comprise the vast majority of all devices which have been implemented clinically.

Standing and locomotion in paraplegia. Studies on paraplegic standing and locomotion are in progress in at least 17 laboratories worldwide including Austria, England, France, Israel, Japan, Netherlands, Scotland, U.S.A., Wales and Yugoslavia. Cybulski, et al., (1984), reviewed development in this application through 1984. Most subjects involved in the studies have had complete thoracic lesions between T6 and T12. Kralj and Bajd (1983) developed a surface stimulation technique which is employed in many laboratories. Using two channels of stimulation on each leg, both standing and reciprocal walking have been demonstrated. One channel directly activates the knee extensor muscles; the second activates a flexion withdrawal of the hip, knee, and ankle by activation of the peroneal, sural, or saphenous nerves (usually the former site is used). Standing is achieved by activation of the knee extensors and upper body lifting. A stride is taken by maintaining stimulation of the knee extensor with one leg and initiating the flexion reflex in the opposite. When the hip is flexed, the knee extensor is activated and maintained through the stance phase. Upon double limb support the subject moves the walking support and triggers the movement in the opposite limb. Trunk stability during stance is achieved by hyperextension of the hips, forming a C-curve posture. Walking is assisted with either a rolling or reciprocating walker or crutches, which contain switches to supply the trigger command inputs to initiate stimulation. A similar technique of activation is used in most laboratories. Generally a function (e.g. Stand, Walk, Climb) is selected by the user and then he initiates the action which is subsequently indicated by a second presentation on the display (e.g. Left Leg). This triggers a sequence of stimulus trains which have been preprogrammed into the device, to activate the muscles to the proper level and in the proper sequences to move the limb through the desired trajectory.

The surface stimulation technique has been used extensively at many centres, since it utilises a noninvasive stimulation technique and activates the minimal number of muscle groups required for walking with the fewest number of channels of stimulation. Recently in Ljubljana, Yugoslavia, a single channel implantable stimulator has also been implanted near the peroneal nerve in a paraplegic subject, with the flexor pattern elicited with this technique as well. Petrofsky has studied walking with subjects wearing external electrodes on up to 12 muscles per leg and external goniometers at the hip, knee, ankle, and shoes with pressure sensors for feedback signals. This system has been reduced to a clinically simpler system by incorporating a reciprocating orthosis and electrodes for stimulation of quadriceps and iliacus incorporated in a wearable garment.

Holle and Thoma (1984) have implanted multichannel receiver-stimulators, with electrodes applied to the femoral and infragluteal nerves for knee extensor (quadriceps) and hip extension (gluteus maximus). Reciprocal walking has been achieved. Walking studies using implantable devices reported by Brindley have apparently not been expanded. 
Marsolais, et al., (1983) has utilised the percutaneous electrode technique to access up to 16 muscles of each extremity, as well as using three channels of surface electrodes for trunk support. Movement provided is hip flexion, extension, and abduction, knee flexion and extension, and ankle plantar and dorsiflexion. Subjects have demonstrated the ability to stand, walk on level ground and slight ramps, climb and descend stairs, and step sideways. Figure 2 is a photograph of one subject presently involved in the research program.

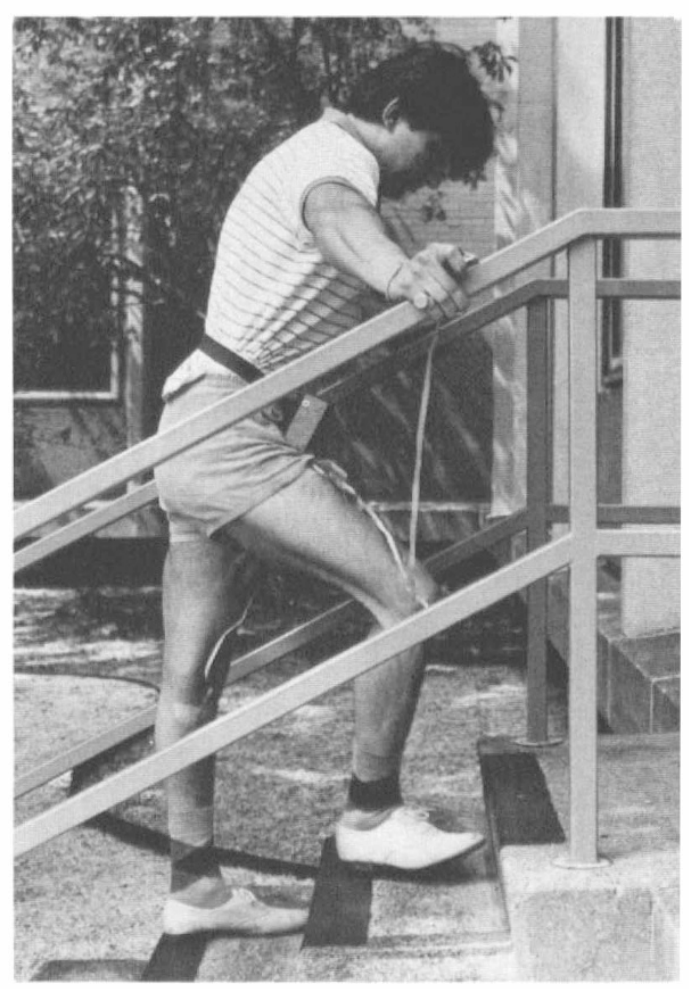

Figure 2 Complete paraplegic subject demonstrating stair climbing with implanted electrode system. (Photo compliments of Dr E. B. Marsolais, Veterans Administration Medical Center and Case Western Reserve University).

This system uses preprogrammed sequences of stimulation to produce each movement trajectory. These are selected from the menu sequence displayed on a liquid crystal display of the stimulator unit. Most movements have utilised open loop control, but feedback control has been incorporated in the knee and hip position.

Incomplete injuries have been studied most extensively by Andrews in Glasgow and Bajd in Ljubljana. The residual voluntary function retained is different and not predictable from one individual to the next because of the nature of the injury. In general, subjects have had considerably more extensive involvement in one extremity than the other, and efforts have been focused on the more involved side. Because many of these subjects retain some sensory 
function on the involved limb, they may be aware of limb placement and joint stability. The approach has been to utilise surface peroneal stimulation alone or combined with stimulation of the knee extensor to initiate and maintain a synthetic gait pattern. Andrews also utilised a sensor in the calf strap of an anklefoot orthosis for feedback control in regulation of the knee extension moment. This ensured that the knee did not collapse during stance.

The clinical acceptance of synthetic walking systems will be measured, to a large extent, upon their ease and reliability in use and their efficiency. At the present time only limited experience on small populations of subjects have been obtained at any single centre. While the results to date demonstrate impressive function, the objective is to reduce the energy expenditure that is required during walking, while providing security that malfunction will not result in a fall and potential fracture. Many approaches have been proposed: closed loop regulation to ensure repeatable function; postural switching to share activity among alternative muscle groups; external orthoses to limit movement and support weight; implanted systems to minimise external harnessing and provide activation of deep muscle groups; command signals which are synchronously generated by activities of the intended gait; etc. While FNS walking systems to this day have had limited applicability, as the results of experimental research are applied in clinical trials, the resolution of some of the most fundamental issues will be resolved. Clinical studies must demonstrate that the technique allows safe functional usage. Potential problems such as joint effusion and degeneration, pressure sores, and bone fracture must be identified at an early time so as to not compromise the user. While the use of FNS for functional walking is in its infancy, the basic techniques of stimulation and control are now developed to a level where their clinical applicability may now begin to be realised. The clinical demonstrations performed to date illustrate substantially enhanced function which is unavailable through alternative sources. Despite the many potential problems to be encountered and unknowns to be resolved in applying these techniques of FNS to provide synthesised walking, the future of this technique appears very promising.

Functional control of the upper extremity. Studies in the upper extremity have focused on development of grasp-release function. Both surface and implanted techniques are employed, but it appears that the isolated activation of quite small muscles is better suited to implanted techniques. Studies performed in our laboratories have focused on providing controlled prehensionrelease in the $\mathrm{C} 5$ and $\mathrm{C} 6$ tetraplegic using implanted electrodes. These subjects generally have bilateral loss of distal muscle function, but voluntary control of the shoulder and elbow flexion. Some subjects may have some voluntary wrist extension. Elbow extension, wrist flexion, and prehension-release is absent. Sensation in the hand is absent or severely impaired.

Neuroprostheses for restoration of controlled prehension-release have been implemented in 26 subjects and undergoing various stages of outpatient clinical trials for 7 years. The neuroprosthesis provides control of two types of grasp; palmar prehension (or three jaw chuck pinch) and lateral prehension (or key grip). Generally eight muscles are used, four or five for each grasp. The subject proportionally controls both the position of his fingers and thumb and the grasp force by a single command control source, generally the position of the shoulder 


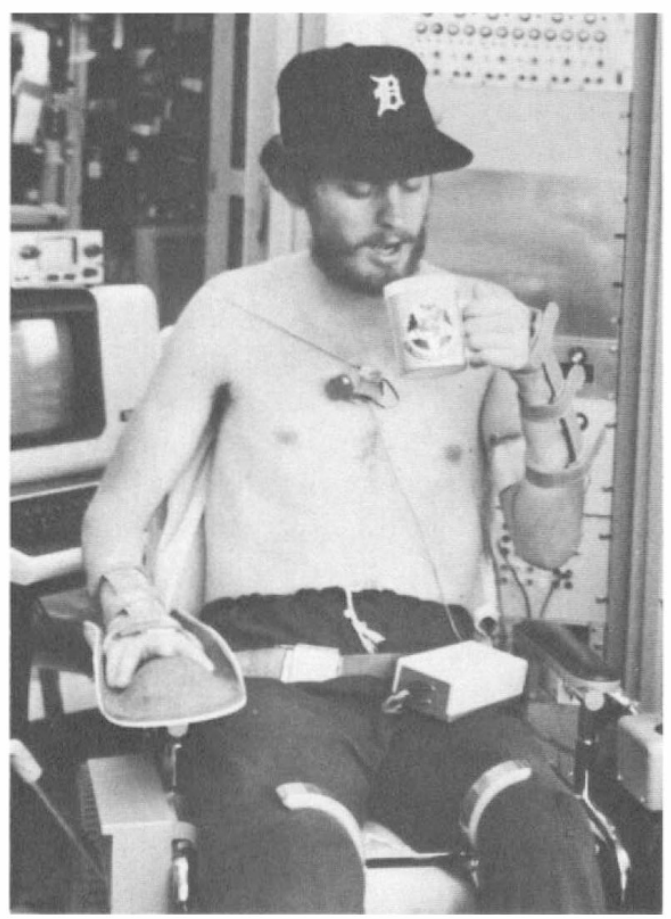

Figure 3 Complete cervical level tetraplegic with FES system for restoration of grasp-release function.

opposite to the limb being stimulated. Figure 3 shows one of the subjects involved in the evaluation of this system. The clinical system operates as an open loop system, with the visual feedback used to ensure adequate grasp on an object.

Chronically indwelling percutaneous electrodes are used for implantation of muscles providing finger and thumb flexion and extension and thumb abduction and adduction. Surgical reconstruction has been performed on the hand in three subjects to enhance the function provide with FNS through stabilisation of the interphalangeal joint of the thumb and synchronisation of the fingers in flexion and extension movement by tenodeses of the Flexor digitorum superficialis, Flexor digitorum profundus, and Extensor digitorum.

Performance of subjects using the FNS system has been evaluated in activities of daily living, including tests of isolated basic tasks, co-ordinated tasks, and integrated tasks. Basic tasks performed by the subjects include grasp release or utensils, books, writing instruments, telephone, cups, etc. Integrated tasks include pouring, washing, diskette handling, teeth brushing. Some subjects demonstrated the ability to perform advanced tasks such as threading a needle and self-catheterisation. Tasks that involved transitions (i.e. acquiring an object, position the object, holding for use, and replacing the object) were able to be accomplished regularly by subjects using FNS. The functional performance demonstrated by the subjects provides them with independence of hand function that was not achieved with the wrist hand orthosis which was available for 
alternative use. With FNS, they were able to perform more tasks independently and lost no abilities as a result of implementing the FNS system. We believe these results demonstrate a major advance in the treatment of the highlevel tetraplegic hand.

Implantable stimulator devices also have been used in upper extremity applications by Pasniczek in Warsaw and more recently in our laboratory by Keith and in that of Brindley (D. Rushton, personal communication).

Control of elbow extension has been demonstrated in the C5 tetraplegic in our laboratory. Stimulation of the lateral and medial heads of the triceps muscle was regulated with the position of the arm in abduction. Thus, as the subject reached to greater angles above his head, the stimulation level was increased to provide sufficient force to fully extend the elbow. If an intermediate position of the elbow was desired, the subject could reach that position by voluntary contraction of his elbow flexors. Combining the hand system with that of the elbow is expected to further enhance independent function.

\section{Advanced FNS systems}

More advanced FNS systems which are under development incorporate closed loop feedback and substitutional sensory feedback. Closed loop feedback systems internally correct for differences between the desired action and the actual action generated by the stimulation (e.g. joint position or contact force). Thus, more repeatable movement can be generated since there is internal compensation for changes such muscle activation properties, (e.g. fatigue), or external disturbances (e.g. gravity or an object impeding movement). The control systems which perform the corrections must function over a wide range of conditions, and have been extensively researched by Crago and Chizeck (1985). At present, satisfactory performance of the controllers and improvement in the actual clinical function has been demonstrated in restricted conditions of the laboratory environment for regulation of motions limited complexity in humans.

Substitutional sensory feedback systems augment function of the FNS system by providing the user with cutaneous sensory cues which are related to the movement or force in the limb. This is important, since the user generally has a sensory deficit in his extremities, and must commit substantial conscious effort toward monitoring the elicited movement to insure that it is sufficient to perform the intended function. Szeto and Riso (1987) have recently reviewed this field. Riso has studied paradigms for generating cutaneous sensation by subcutaneously placed electrodes. Subjects have demonstrated the ability to accurately recognise cutaneous stimuli with high resolution at adequate rates and with stability of the response over time. Some aspects of these techniques have been incorporated in the upper extremity system for control of grasprelease, and more extensive integration of this technique into the clinical systems may be anticipated to lead to more confident and higher quality performance with the FNS system.

Sensors are required to provide the sources of information in both closedloop feedback and sensory feedback. Crago et al., (1986) has recently reviewed the sensor requirements for FNS. For the upper extremity, joint position and contact force information is required. For the lower extremity, joint position, foot-floor contact, and crutch reactions are needed. At present, few sensor 
systems meet the investigational needs, although substantial improvement on all of these sensors has been made. For example, in the upper extremity a single element force sensor has been developed which can be worn over the contact area of the thumb and fingers, and a two degree of freedom joint position sensor is used. These sensors are intended primarily for laboratory evaluation, but ultimately the type of information they provide must be available from internal sensors which are not unencumbering to the user. Development of sensor technology thus may be expected to be a substantial effort in implementation of more advanced FNS systems.

\section{Conclusion}

The future development of functional electrical stimulation may be expected to proceed along several pathways; technical, biological sciences, and clinical sciences. Technical development will include the development of improved sensors, stimulators, electrodes, and so forth. The natural progression of technical development toward smaller, lower power processors with greater computational capabilities will assist the development of clinical hardware. Development of the biological sciences will provide far greater understanding of neural events, such as mechanisms of neural excitation, and information available from chronic neural recordings in the peripheral and central nervous system. This information will be used to develop more selective electrodes, to provide alternative sources of feedback and command control information, etc. Clinical efforts will be the beneficiary of these findings, as we learn to apply increasingly sophisticated neural prostheses to provide increasingly more refined movements.

The challenge of incorporating neural prosthetic technology to solve the clinical problems is substantial. Clinicians and scientists, with the users, must collaborate closely in defining reasonable clinical objectives, identifying the approach to specific problems, and in implementing the solutions. FES should not be expected to resolve complex disorders of the central nervous system in isolation of other treatment modalities. Rather, it must be used in combination with these modalities such as therapy, surgery, orthotics, and other assistive aids. Both clinical and basic science advances in FES are introduced regularly. These results demonstrate that the challenge is being met and provide good reason for cautious optimism regarding the significance of FES in rehabilitation of the spinal cord injured.

\section{Acknowledgement}

This research was supported in part by the National Institute of Health, Neural Prosthesis Program (NO1-NS-3-2334), the National Institute on Disability and Rehabilitation Research (9008300118) and the Veterans Administration Research and Development Service.

\section{References}

Benton LA, BAKer LL, Bowman BR, et al. Functional Electrical Stimulation-A Practical Clinical Guide. Rancho Los Amigos REC, Downey, CA

Crago PE, Chizeck HJ, Neuman MR, et al. Sensors for use in Functional Neuromuscular Stimulation. IEEE Transactions on Biomedical Engineering 33:256-268 
Cybulski GC, Penn RD, Jaeger RJ 1984 Lower Extremity Functional Neuromuscular Stimulation in Cases of Spinal Cord Injury. Neurosurgery 15:132-146

Hambrecht FT, Reswick JB 1977 Functional Electrical Stimulation: Applications in Neural Prostheses. New York: Marcel Dekker.

Handa Y, IChie M, Handa T, et al. 1985 Control of the Paralyzed Hand by a ComputerControlled FES System. Proceedings of the Seventh IEEE-EMBS conference 322-326

Holle J, Frey M, GRUBer H, et al. 1984 Functional Electrostimulation of Paraplegics:

Experimental Investigations and First Clinical Experience with an Implantable Stimulation Device. Orthopaedics 7(7):1145-1160

KRALJ A, BAJD T, TURK R 1983 Gait restoration in paraplegic patients: A Feasibility Demonstration using multichannel surface electrode FES. Fournal of Rehabilitation Research and Development 20(BPR 10-38):3-20.

Marsolais EB, Kobetic R 1983 Functional Walking in Paralyzed Patients by Means of Electrical Stimulation. Clinical Orthopaedics 175:30-36

Marsolais EB, Kobetic R 1986 Implantation Techniques and Experience with Percutaneous Intramuscular Electrodes in the Lower Extremities. Fournal of Rehabilitation Research and Development 23:6:1-8

Mortimer JT 1981 Motor prosthesis in Handbook of Physiology, Section I: The Nervous System, Vol. 2, Part I, Chap. 5, Brooks VB (ed.). Am. Physiological Soc., Bethesda, MD 155-187

Petrofsky JE, Phillips CA 1985 Closed-loop Control of Movement of Skeletal Muscle. CRC Critical Reviews in Biomedical Engineering 13:35-96

Szeto AYJ, Riso RR 1987 Sensory Feedback Using Electrical Stimulation. In: Smith RV, LeSLIE JH (eds), Rehabilitation Engineering. CRC Press, Boca Raton, FLA

Wilhere GF, Crago PE, Chizeck HJ 1985 Design and Evaluation of a Digital Closed-Loop Controller for the Regulation of Muscle Force by Recruitment Modulation. IEEE Transactions on Biomedical Engineering 32:668-676 